\title{
Liquid-crystal electric tuning of a photonic crystal laser
}

\author{
Brett Maune, ${ }^{a}$ Marko Lončar, Jeremy Witzens, Michael Hochberg, Thomas Baehr-Jones, \\ Demetri Psaltis, and Axel Scherer \\ Department of Electrical Engineering and Applied Physics, California Institute of Technology, Pasadena, \\ California 91125
}

\section{Yueming Qiu}

In Situ Technology and Experiments Systems Section, Jet Propulsion Laboratory, California Institute of Technology, Pasadena, California 91109

(Received 2 December 2003; accepted 19 May 2004)

\begin{abstract}
An electrically tuned nematic liquid-crystal (LC) infiltrated photonic crystal (PC) laser is demonstrated. This PC laser represents an emerging class of nanoscale optical adaptive devices enabled by the convergence of nonlinear optical materials, electronics, and fluidics that promise increased functionality and utility over existing technologies. A LC cell is constructed by encasing the PC laser between two indium tin oxide glass plates, which serve as the modulating electrodes. Applying a voltage across the cell realigns the LC, modifies the laser cavity's optical path length, and blueshifts the lasing wavelength. (C) 2004 American Institute of Physics.
\end{abstract}

[DOI: $10.1063 / 1.1772869$ ]

Microfabricated photonic crystals (PCs) enable the microscopic manipulation of light within two- and threedimensionally (2D) periodic geometries, ${ }^{1,2}$ and have been used to realize efficient nanoscale optical cavities and lasers. ${ }^{3}$ Light emitted from a PC laser, although generated from a semiconductor slab, can be concentrated in the air pores that define the PC. By infiltrating the PC laser with a liquid, this unique feature of $\mathrm{PC}$ lasers enables the efficient interaction of the confined lasing field with the infiltrated material. Here we report on the successful liquid crystal (LC) tuning of 2D PC lasers-devices in which the lasing frequency can be electrostatically controlled with an applied gate voltage.

The resonant wavelength of an optical cavity can be tuned by adjusting the cavity's refractive index, ${ }^{4}$ and hence, its optical path length. Such tuning has previously been accomplished in a 2D PC laser by lithographically controlling the local cavity geometry ${ }^{5}$ or by filling of the holes of the PC with liquids of different refractive index. ${ }^{6}$ Until now, it has been difficult to actively tune the emission frequency of PC lasers by deliberately changing the refractive index of the material inside of the cavity. To electrostatically tune PCs, infiltration of LCs into the pores of such structures was proposed several years ago. ${ }^{7}$ Since then, several groups have demonstrated dynamic thermal tuning of PCs with infiltrated $\mathrm{LCs}^{8-10}$ and more recently electrical tuning via LCs has been reported. ${ }^{11-13}$ In this letter we present our work on electrically tuning 2D PC lasers by controlling the orientation of infiltrated nematic LC.

Liquid crystals offer a relatively large birefringence and correspondingly large refractive index tuning ranges. Before LC tunable PC lasers can be constructed, several design constraints need to be addressed. Infiltrating the PC with a liquid decreases the refractive index contrast of the system. This results from the ambient refractive index change from that of air $(n=1)$ to that of a LC $(n \sim 1.45-1.7)$. The lower refractive index contrast in turn narrows the photonic bandgap and decreases both in-plane and vertical confinement of the light,

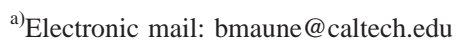

reducing the cavity's quality factor $(Q)$. Thus, the relatively high refractive indices of LCs demand a significant redesign of the laser cavity geometry. Moreover, their large birefringence can scatter light if the LC is not aligned uniformly. Therefore, the nematic LC chosen for this study (MLC-6815 from Merck) has a deliberately low refractive index $\left(n_{e}\right.$ $=1.519, n_{o}=1.467$ at $589 \mathrm{~nm}$ ) and a modest birefringence $(0.05$ at $589 \mathrm{~nm})$. The $Q$ of the PC cavity can then be sufficiently optimized to enable lasing even after infiltration of the PC.

To accommodate the low refractive index contrast, a high $Q$ PC cavity design was used. ${ }^{14}$ A scanning electron microscopy image of the PC laser cavity is shown in Fig. 1. The device geometry optimized the $Q$ of one of the dipole modes supported by the laser cavity to approximately 2000 , even when operated within an ambient refractive index of $n \sim 1.5$. Photonic crystal lasers using this design were defined within InGaAsP quantum well material and fabricated using a combination of electron beam lithography and dry

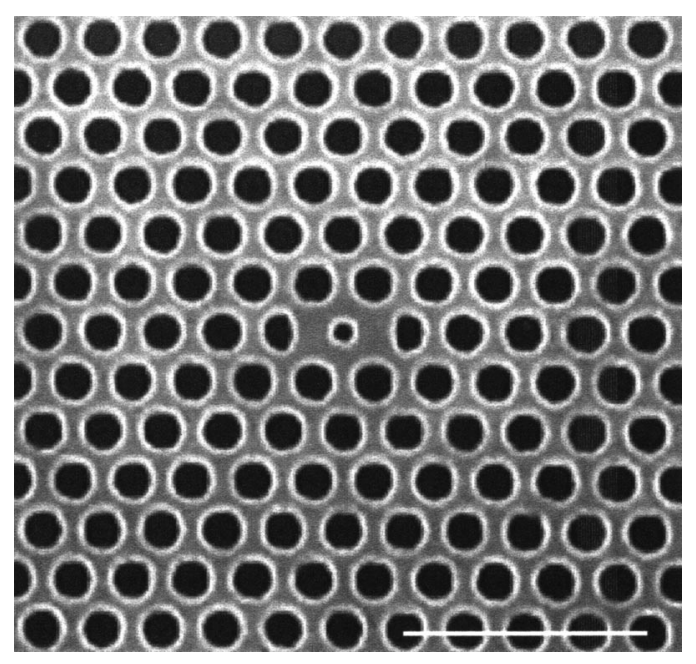

FIG. 1. Scanning electron micrograph of fabricated 2D PC laser. Periodicity of holes is $500 \mathrm{~nm}$, radii of holes are $\sim 165 \mathrm{~nm}$, radius of defect hole is $\sim 100 \mathrm{~nm}$, slab thickness $\sim 320 \mathrm{~nm}$. Scale bar, $2 \mu \mathrm{m}$. 

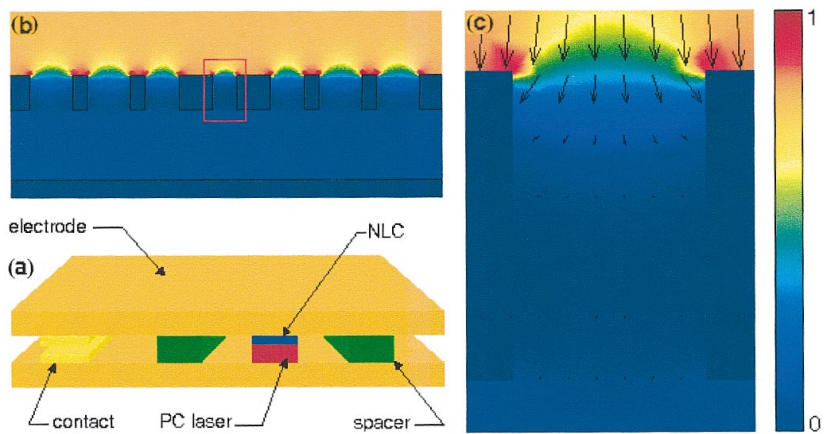

FIG. 2. (Color) Cross section of the PC LC cell with simulated generated electric field. (a) Cross section of PC LC cell. (b) A 2D slice through the PC laser membrane shows the magnitude of the electric field generated by the electrodes. Field magnitude is denoted by normalized color scale from 0 to 1. (c) A close-up of the generated electric field in the defect hole. Arrow direction and length denote field direction and amplitude, respectively.

etching. The details of the laser fabrication procedure have been previously described. ${ }^{15}$

To construct the LC cell around the lasers, we attached the laser sample along with two spacers to an indium tin oxide (ITO) transparent conducting plate with polymethylmethacrylate. Next, a drop of the LC was placed on the PC sample and then the 0.3-nm-thick top ITO plate was glued to the spacers. The spacers were sufficiently thick to obtain an estimated $15 \mu \mathrm{m}$ gap between the sample and top ITO electrode, which was chosen to minimize absorption at $1.55 \mu \mathrm{m}$, exhibiting $92 \%$ transmission at that wavelength. The LC was then heated above the clearing point $\left(67^{\circ} \mathrm{C}\right)$ on a hotplate at $90^{\circ} \mathrm{C}$ for $5 \mathrm{~min}$ to help ensure complete infiltration into the PC. Finally, electrical leads were attached to the ITO plates with conductive tape to complete the LC cell. A cross section of the fully assembled LC cell appears in Fig. 2(a).

Samples were measured using a microphotoluminescence setup. ${ }^{15}$ Lasing after LC infiltration was confirmed by the recording of a characteristic output power versus input curve ( $L-L$ curve). The lasing mode was also linearly polarized in agreement with finite-difference time-domain (FDTD) simulations. The PC lasers were pumped with $4.4 \mathrm{~mW}$ from a semiconductor laser diode at $830 \mathrm{~nm}$ with a pulse length of $15 \mathrm{~ns}$ and a periodicity of $1.5 \mu \mathrm{s}$. Pump light was transmitted through the top plate and the LC onto the PC slab. During the optical pumping of the PC lasers, the effective refractive index of the cavities was changed by application of an electrostatic field between the top ITO electrode and the InP wafer substrate. The expected tuning range strongly depends on the LCs refractive indices and the birefringence at the lasing wavelength $\lambda \sim 1.55 \mu \mathrm{m}$, the spontaneous (zero-field) LC orientation, and the electric field pattern generated by the electrodes. To establish a base line for the laser tuning range, the $\mathrm{LC}$ is assumed to initially be randomly orientated, and the influence of the applied voltage for realignment of the LC is modeled using electrostatic simulations of the generated electric field patterns [Figs. 2(b) and 2(c)]. These simulations indicate that the LC in the top cladding can be aligned vertically, but the electric field is sharply damped in the holes of the PC due to screening by the conducting PC membrane. The lasing mode under investigation not only samples the refractive index in the holes of the PC, but also has an evanescent field in the top cladding layer and is influenced by the refractive index in that region. Therefore, tuning of the cavity can be achieved by changing the refractive index in the holes or in the cladding layers.

We demonstrate LC tuning of the PC laser by recording the lasing wavelength as a function of the voltage applied across the LC cell. Our electrostatic simulation results predict that the LC in the top cladding should align vertically to the surface, lowering $n_{\text {top }}$ from 1.485 to 1.467 and blueshifting the lasing wavelength. ${ }^{16}$ When the voltage is varied from 0 to $20 \mathrm{~V}$, we observe a corresponding blueshift in Fig. 3(a). The wavelength shift starts at $\sim 4 \mathrm{~V}$, the threshold voltage of the LC, signaling the existence of a Freedericksz transition. ${ }^{17,18}$ A maximum tuning of $1.2 \mathrm{~nm}$ is observed at $20 \mathrm{~V}$ and the tuning appears to be independent of the dc field polarity. The slight hysteresis and higher-than-expected threshold voltage in the tuning data may be attributed to charging and impurity effects in the LC [Fig. 3(b)]. The tuning range of the lasers is strongly influenced by the zero-field LC orientation. The tuning range is dependent upon the effective refractive index difference from the zero-field state to the saturated state. The refractive index of the saturated state
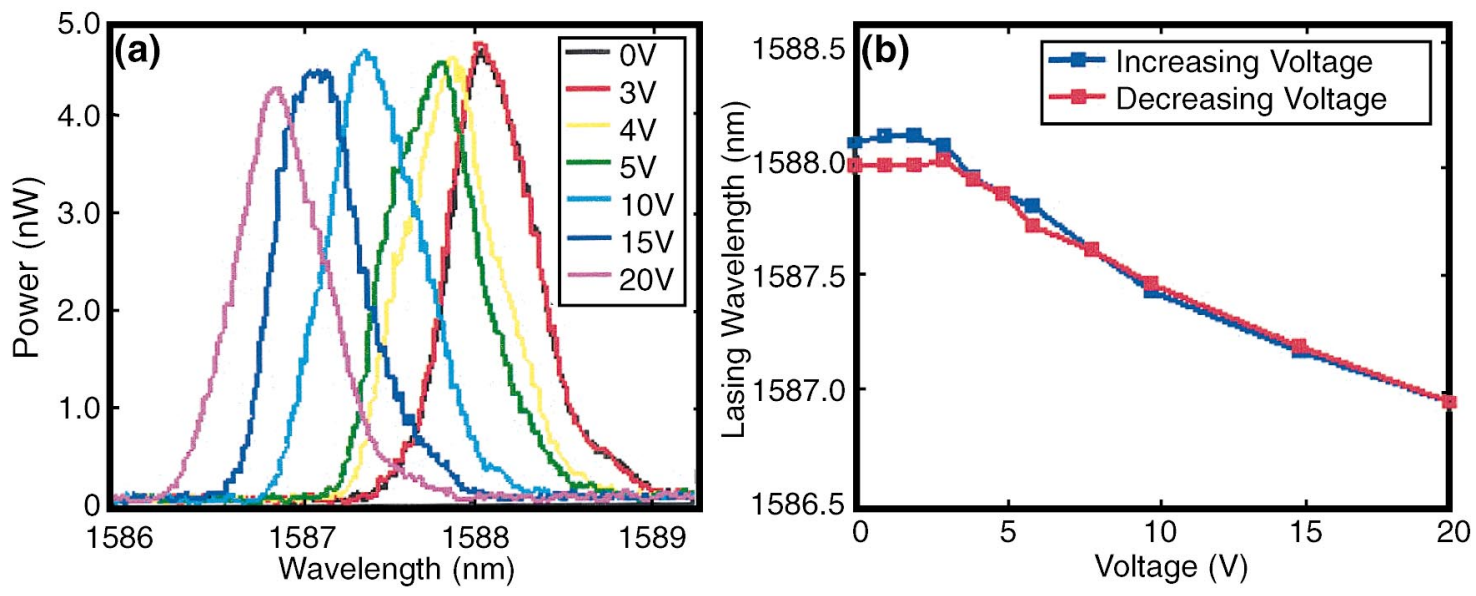

FIG. 3. (Color) Demonstration of laser tuning via LC realignment. (a) Laser spectra taken with an applied voltage ranging from 0 to $20 \mathrm{~V}$ across the LC cell. The threshold for tuning is $\sim 4 \mathrm{~V}$, coinciding with the measured threshold voltage for the LC. A maximum blueshift of $1.2 \mathrm{~nm}$ was measured with an applied $20 \mathrm{~V}$. (b) Lasing wavelength vs applied voltage for a voltage ramp cycle. Although the tuning rate lessens at higher voltage, the data suggest saturation was not even reached at $20 \mathrm{~V}$, and further tuning may be possible with stronger fields. The tuning is reversible but demonstrates a slight hysteresis at low fields which may be due to charging or impurity effects. The marker box height represents the upper bound of the uncertainty in the lasing wavelength obtained from the spectra.

Downloaded 14 Dec 2005 to 131.215.225.171. Redistribution subject to AIP license or copyright, see http://apl.aip.org/apl/copyright.jsp 
is always the same, $n_{0}$, whereas the zero-field refractive index is not. To see this, first consider that the lasing mode is predominantly linearly polarized in the plane of the PC and, hence, features a well defined polarization axis. Second, polarization interference microscopy results suggest the LC spontaneously forms domains oriented homogenously (parallel) to the PC slab. Therefore one expects variablity in the laser tuning range dependent upon the orientation of the domains with respect to the lasing mode's polarization axis. Considering the saturated state always leaves the LC oriented perpendicular to the polarization axis, a maximum tuning is expected when a LC domain is initially oriented such that the directors are parallel to the lasing mode axis and a minimum tuning when oriented perpendicular. The observed distribution in the tuning range data is in agreement with this hypothesis.

Surface anchoring experienced by the $\mathrm{LC}$ also affects the laser tuning characteristics. The tuning behavior at strong fields suggests surface anchoring heavily influences the alignment dynamics of the LC in the vicinity of the PC membrane. The protracted transition to a saturated aligned state further indicates the LC spontaneously aligns homogenously to the PC membrane. As the electric field is steadily increased, LC molecules progressively closer to the membrane surpass the free energy barrier and align with the field. This progressive alignment may explain the continued tuning of the lasers even far above the saturation voltage. By inhibiting the realignment of the LC near the PC-where the lasing mode's evanescent field is strongest-the surface anchoring decreases the tuning range of the lasers. Although overall tuning is limited, a benefit of the surface anchoring is that it enables the lasers to be continuously tuned over a range of wavelengths with angström or even sub-angström accuracy.

One of the outstanding questions is the degree of LC alignment in the PC holes. The electrostatic simulations indicate the field is screened from the holes and little controlled alignment is expected. FDTD simulations predict a much larger tuning, in excess of $6 \mathrm{~nm}$, if both the holes and top cladding are aligned from a random state. The observed tuning is considerably smaller, indicating negligible alignment in the holes. Liquid crystals in such confined geometries can experience a relatively strong surface anchoring and feature a homeotropic or escaped radial orientation amongst others. ${ }^{8,19,20}$ If the surface binding energies could be reduced, then the alignment in the top cladding layer might propagate through the holes and dramatically increase the tuning of the laser.

The demonstration of a LC infiltrated electrically tuned PC laser is significant in several respects. By incorporating various device optimizations including infiltrating the cavity with LCs featuring larger birefringence, increasing the effectiveness of LC alignment with in-plane electrodes or alignment layers, and by controlling the alignment in the holes of the $\mathrm{PC}$, the tuning range of the laser can potentially be increased beyond $20 \mathrm{~nm}$ and qualify the laser to fulfill a variety of technical applications. Such a laser provides an excel- lent and unique opportunity to probe the behavior of LCs confined in nanoscale geometries and enables the simultaneous study of the LCs nonlinear optical interaction with an intense optical field. More broadly, the successful integration of electronics and fluidics with PC lasers demonstrates the flexibility these structures possess and suggests even further functionalization is achievable. Furthermore, the lasers can be infiltrated with materials featuring wide ranging linear and nonlinear optical properties and therefore facilitates the study of an enormous assortment of optical phenomenon within a nanoscale intense optical field previously not possible.

The authors would like to thank Mark Adams, David Barsic, Rhys Lawson, Xu Wang, and Tomoyuki Yoshie for valuable discussions. Funding for this work by Jag Shah from DARPA under the CS-WDM program (Contract No. N00421-02-D-3223) is also gratefully acknowledged.

${ }^{1}$ E. Yablonovitch, Phys. Rev. Lett. 58, 2059 (1987).

${ }^{2}$ S. John, Phys. Rev. Lett. 58, 2486 (1987).

${ }^{3}$ O. Painter, R. K. Lee, A. Scherer, A. Yariv, J. D. O'Brien, P. D. Dapkus, and I. Kim, Science 284, 1819 (1999).

${ }^{4}$ B. Maune, R. Lawson, C. Gunn, A. Scherer, and L. Dalton, Appl. Phys. Lett. 83, 4689 (2003)

${ }^{5}$ O. Painter, A. Husain, A. Scherer, P. T. Lee, I. Kim, J. D. O'Brien, and P. D. Dapkus, IEEE Photonics Technol. Lett. 12, 1126 (2000).

${ }^{6}$ M. Loncar, A. Scherer, and Y. Qiu, Appl. Phys. Lett. 82, 4648 (2003).

${ }^{7}$ K. Busch and S. John, Phys. Rev. Lett. 83, 967 (1999).

${ }^{8}$ S. W. Leonard, J. P. Mondia, H. M. van Driel, O. Toader, S. John, K. Busch, A. Birner, U. Gösele, and V. Lehmann, Phys. Rev. B 61, R2389 (2000).

${ }^{9}$ G. Mertens, T. Roder, R. Schweins, K. Huber, and H. S. Kitzerow, Appl. Phys. Lett. 80, 1885 (2002).

${ }^{10}$ C. Schuller, F. Klopf, J. P. Reithmaier, M. Kamp, and A. Forchel, Appl. Phys. Lett. 82, 2767 (2003)

${ }^{11}$ D. Kang, J. E. Maclennan, N. A. Clark, A. A. Zakhidov, and R. H. Baughman, Phys. Rev. Lett. 86, 4052 (2001).

${ }^{12}$ Y. Shimoda, M. Ozaki, and K. Yoshino, Appl. Phys. Lett. 79, 3627 (2001).

${ }^{13}$ R. Ozaki, T. Matsui, M. Ozaki, and K. Yoshino, Appl. Phys. Lett. 82, 3593 (2003).

${ }^{14}$ M. Loncar, M. Hochberg, A. Scherer, and Y. Qiu, Opt. Lett. 29, 721 (2004).

${ }^{15}$ M. Loncar, T. Yoshie, A. Scherer, P. Gogna, and Y. Qiu, Appl. Phys. Lett. 81, 2680 (2002).

${ }^{16} \mathrm{By}$ infiltrating the lasers with refractive index-matched fluids and comparing the lasing redshift with that of the LC infiltrated lasers, we determined the LCs refractive index to be the same at IR wavelengths (within the experiment's resolution) as at visible wavelengths. Therefore, in this work we assumed the LCs refractive indices at the lasing wavelengths to be those specified at visible wavelengths.

${ }^{17}$ Polarization interference microscopy was used to verify the existence of a Freedericksz transition in the LC cell. This method yielded an observed threshold voltage of $\sim 4 \mathrm{~V}$, in agreement with the onset of tuning of the PC laser.

${ }^{18}$ L. Blinov, in Handbook of Liquid Crystals, edited by D. Demus, J. Goodby, G. W. Gray, H. W. Spiess, and V. Vill (Wiley-VCH, New York, 1998) Vol. 1, Chap. 9.

${ }^{19}$ G. P. Crawford, M. Vilfan, J. W. Doane, and I. Vilfan, Phys. Rev. A 43, 835 (1991).

${ }^{20}$ G. P. Crawford, D. W. Allender, J. W. Doane, M. Vilfan, and I. Vilfan, Phys. Rev. A 44, 2570 (1991). 